\title{
Role of Magnetic Resonance Imaging with Diffusion Sequences in Assessment of Multiple Sclerosis in Brain Versus Normally Appearing White Matter
}

\author{
Hesham E. El Sheikh, Ahmed S. Mohamed, Ehab M. Sharweda
}

\begin{abstract}
Department of radiology, Benha faculty of medicine, Banha University, Egypt.

Correspondence to: Ehab M. Sharweda, Department of radiology, Benha faculty of medicine, Banha University, Egypt.
\end{abstract}

Email:

ehab.sharweda@yahoo.com

Received: 15 December 2020

Accepted: 10 February 2021

medical sheet and MR examination including: Conventional MR examination and Diffusion Tensor imaging. Technique was performed using a standard 1.5 Tesla unit. Results: The study showed that DTI can reveal changes in NAWM in MS cases before visible sizable plaques can be detected by conventional MRI.

Key Words: Multiple sclerosis, Magnetic resonance imaging, Advanced MRI techniques, Diffusion tensor imaging.

\begin{abstract}
:
Background: Magnetic resonance imaging (MRI) is a very vital tool to diagnose and monitor multiple sclerosis (MS). Standard MRI measures lack of pathological specificity and are weakly correlated with MS clinical manifestations. Advanced MRI techniques together with diffusion studies square measure up the understanding of the mechanisms underlying tissue injury, repair and functional adaptation in MS but, they need careful standardization. It doesn't only enhance the understanding of the pathophysiology and evolution of disease, but also to generate research hypotheses, monitor treatment, increase costeffectiveness and power of clinical trials. Aim of the Work: The aim of this study is to evaluate the role of DTI in assessment of MS versus the normally-appearing white matter (NAWM). Patients and Methods: The study included 50 patients, 42 females and 8 males having MS (between 20 and 40 years of age) referred from Neurologists to Radio-diagnosis Department at Benha University hospitals with 5 age-matched control subjects (during the period between Aug. 2018 and Jan. 2020). Each patient included in the study was subjected to full history taking, reviewing
\end{abstract}





\section{Introduction}

Multiple sclerosis is a chronic autoimmune condition of the central nervous system (CNS) characterized by blood-brain barrier (BBB) breakdown, inflammation, myelin damage, and axonal loss. The pathogenesis of MS is unknown; apart from a genetic predisposition and previous virus infections are thought to be relevant. Multiple sclerosis is estimated to affect 2.5 million individuals worldwide. Multiple sclerosis typically presents in young Caucasian adults, with a peak between 20 and 40 years. There is increasing evidence for appearance of first manifestations of MS at childhood and older ages as well. Multiple sclerosis is twice as common in women as in men ${ }^{(1)}$.

Magnetic resonance imaging has become a cornerstone for the diagnosis and monitoring of MS. Its high sensitivity for inflammatory and neurodegenerative processes assessment in the brain has made it the most commonly used tool for the evaluation of MS patients. Besides, MRI has become a powerful tool for treatment monitoring and for the prognostication of disease as well ${ }^{(2)}$.

Magnetic resonance imaging new techniques are challenging the notion that all lesions evolve in this stereotyped pattern, in which focal BBB leakage and perivascular inflammation is the initiating event. There is evidence from some studies using magnetization transfer imaging, giving a measure of tissue integrity, that subtle structural abnormalities in areas of NAWM may precede any detectable changes on Gd-DTPA-enhanced or $\mathrm{T}_{2^{-}}$ weighted scans ${ }^{(2)}$.

Diffusion Tensor Imaging (DTI) is one of the most promising techniques that have been investigated in the study of MS. It quantifies the amount of non-random water diffusion within tissues and provides unique in vivo information about the pathological processes that affect water diffusion as a result of brain microstructural damage. DTI provides a unique method of imaging contrast and quantification that allows better understanding of the pathophysiology of MS-related white matter damage that would also be useful for monitoring disease progression and treatment effects ${ }^{(3)}$.

\section{Aim of work}

The aim of this study is to evaluate the role of DTI in assessment of MS versus the NAWM; thus, assessing its ability to detect early abnormalities at diffusion level. 


\section{Patients and Methods}

This retrospective study included 50 patients 42 females and 8 males having MS (between 20 and 40 years of age) referred from Neurologists to Radio-diagnosis Department at Benha University hospitals with 5 age-matched healthy control subjects (between August 2018 and January 2020). These procedures are approved by local Ethics Committee. All patients provided a written informed consent.

Inclusion criteria:

- Proven to be MS patient.

- Approved to participate in our study.

- Approved to perform DTI examination.

Exclusion criteria:

- Patients with other brain parenchymal pathology, e.g. brain neoplasm.

- Patient with other neurological disorder.

- Patients with contraindication to perform MRI examination (e.g. claustrophobic patients).

- Patient refused to participate in our study.

- Patient refused to do DTI examination.

Each patient included in the study was subjected to:

- Full history taking.
- Reviewing medical sheet whenever available.

- MR examination including:

1.Conventional MR examination

2.Diffusion Tensor imaging.

Technique:

- All patients were briefly instructed about our study and duration of examination.

- Proper preparation (regarding proper clothing and metal disposal) instructions were delivered and checked by the aiding nurse.

- Technique was performed using a standard closed 1.5 Tesla unit.

- A standard head coil was used.

- Patient position: Supine.

- Conventional MRI sequences obtained which consisted of Axial T1WI, Axial T2WI, Axial FLAIR and Sagittal FLAIR

- Following DTI sequence was obtained:

- A single shot, spin-echo echo planar sequence in 25 encoding directions and a diffusion weighting factor of 800 s/mm2. TR 10951, TE 67, matrix 128 x128, FOV 224 X $224 \mathrm{~mm}$, number of excitations 2, slice thickness: 2.0/00 and flip angle 90 (degrees).

- All the diffusion-weighted images were transferred to the workstation 
supplied by the manufacturer.

- Images were post-processed using the Philips software devised for tractography. The maps obtained were:

- FA 2D grey maps.

- Directionally-encoded color FA maps and fused FLAIR/DTI maps.

- Application of multiple (color coded) ROI at both frontal, parietal, occipital normal appearing white matter, brain stem and corpus callosum followed by analysis of the parametric indices results (FA, MD)

\section{Results}

The study included 42 female patients and 8 male patients between 20 and 40 years old (Fig.1). Comparison with fractional anisotropy (FA) and mean MD of control subject's white matter regions was made. Pie charts were done for ratio of cases showing one or more normal appearing white matter region of FA reduction and/or MD affection.

$84 \%$ of the cases showed at least one region of NAWM FA reduction (Fig.2) while 40\% of the examined cases showed increased MD in NAWM (Fig.3).

Previous statistics show that more degree of FA affection was noted as compared to the MD affection of the examined cases. So, FA may be used for early detection of the NAWM affection owing to its sensitivity.

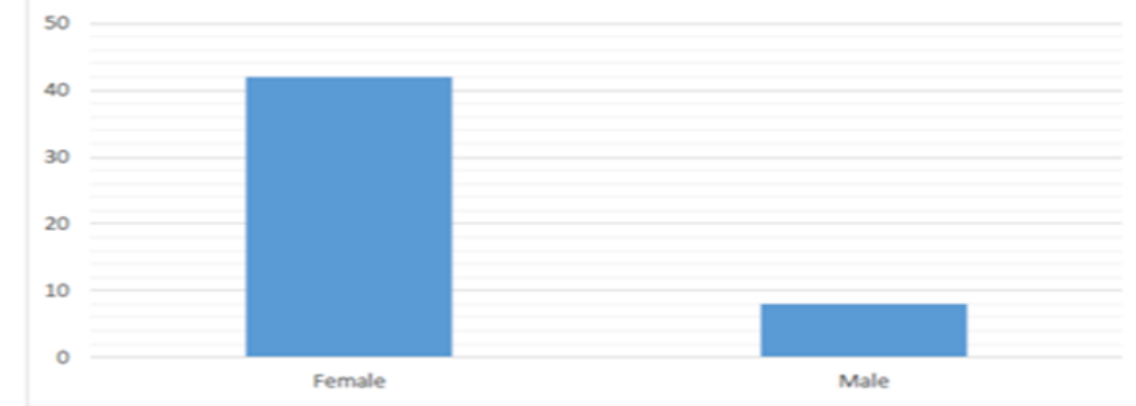

Fig. 1 female/male ratio of total cases 


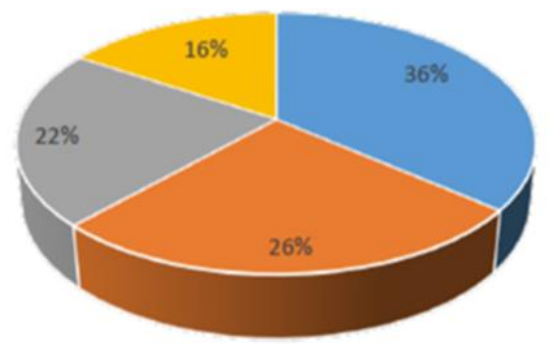

- Cases with one region of FA

reduction

- Cases with two regions of FA

reduction

- hree or more regions of FA

reduction

- Cases with no NAWM affection

Fig. 2 cases with FA affection in relation to total cases

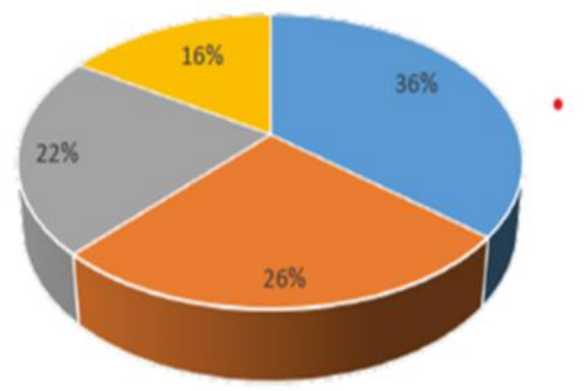

- Cases with one region of FA

reduction

- Cases with two regions of FA reduction

- hree or more regions of FA reduction

- Cases with no NAWM affection

Fig. 3 cases with $\mathrm{MD}$ affection I relation to total cases

\section{Cases}

Case (1)

- Clinical profile: MS male patient of 30 years old with diagnosed MS several years ago and recurrent bouts of clinical symptoms that are resistant to therapy.

- The conventional MRI findings: Irregular patchy areas (plaques) of abnormal white matter signal intensity are seen implicating both cerebral peri-ventricular and subcortical regions (Fig.4).

- DTI findings: Fused FLAIR/DTI axial images revealed heterogeneous cartography
- maps colors of the subcortical and periventricular as well as the brain stem (Fig.5) white matter denoting NAWM affection with parametric indices as seen in table 1 .

- Comment: Significant reduction of the FA values of the NAWM within the right frontal, right occipital and the left pontine regions i.e. not the most affected regions on conventional MRI. 


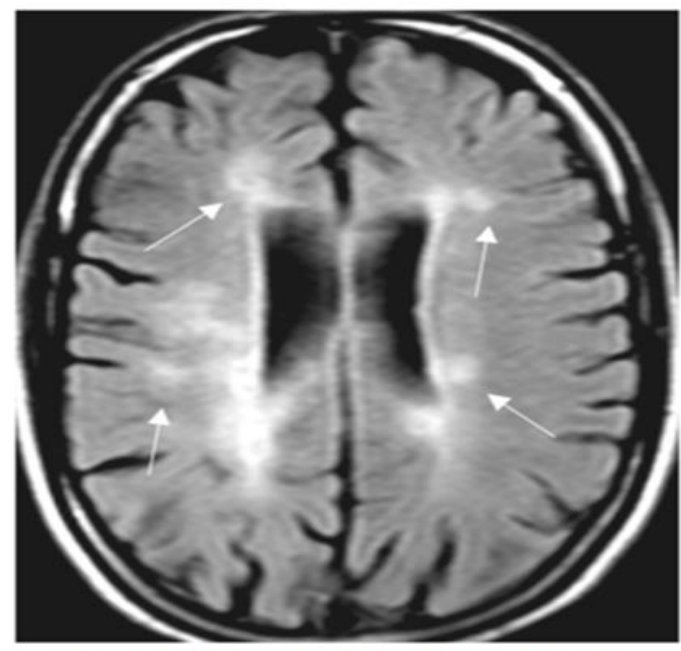

Fig. 4 Axial T2/FLAIR of case (1). MS

plaques are most evident at bilateral periventricular and subcortical regions (white arrows).
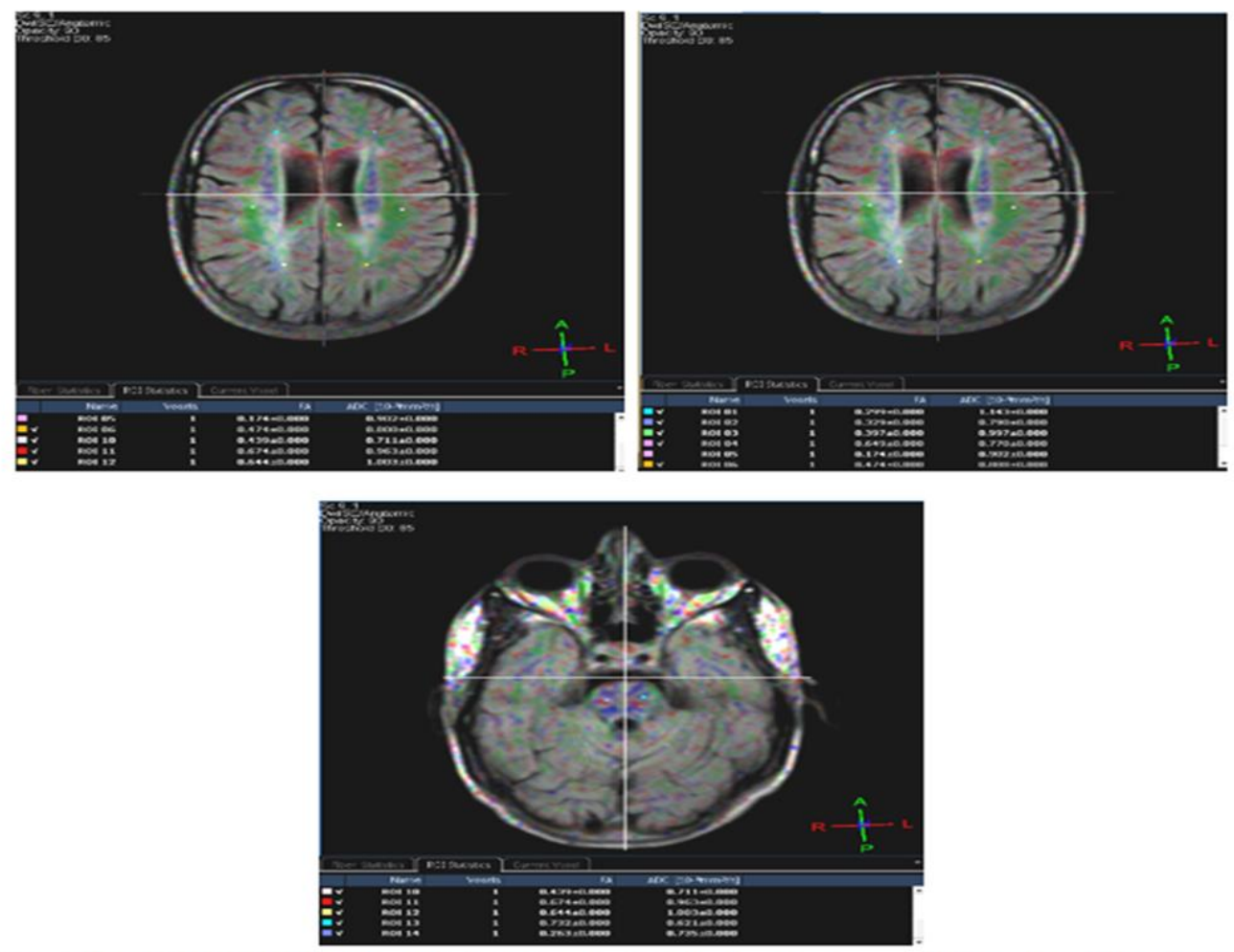

Fig. 5 Fused FLAIR/DTI with multiple ROI at both frontal, parietal, occipital, corpus callosum and brain stem on normally appearing white matter with parametric indices results (FA, MD and ADC) in case (1). 

Table 1: Parametric indices of case (1)

\begin{tabular}{lccc}
\hline White matter region & FA & ADC & MD \\
\hline Right frontal & 0.2 & 1.14 & 0.0033 \\
Left frontal & 0.3 & 0.8 & 0.0035 \\
Right parietal & 0.4 & 1.1 & 0.0028 \\
Left parietal & 0.65 & 0.8 & 0.0027 \\
Right occipital & 0.2 & 0.9 & 0.003 \\
Left occipital & 0.5 & 0.9 & 0.0031 \\
Corpus callosum & 0.6 & 1 & 0.0027 \\
Brain stem & 0.7 & 0.7 & 0.0032 \\
\hline
\end{tabular}

Case (2)

Clinical profile: Known case of MS female patient of 40 years old with recurrent bouts of clinical symptoms and under regular follow up.

The conventional MRI findings: Irregular patchy areas (plaques) of abnormal white matter signal intensity are seen implicating both cerebral peri-ventricular and subcortical regions. They are seen mostly implicating the left parietal region (Fig.6)
DTI findings: Fused FLAIR/DTI axial images revealed evident heterogeneous cartography maps colors of the left parietooccipital white matter (Fig.7) denoting NAWM affection with parametric indices as seen in table 2 .

- Comment: Significant reduction of the FA values of the NAWM within the right occipital and frontal regions i.e. not the most affected regions on conventional MRI.

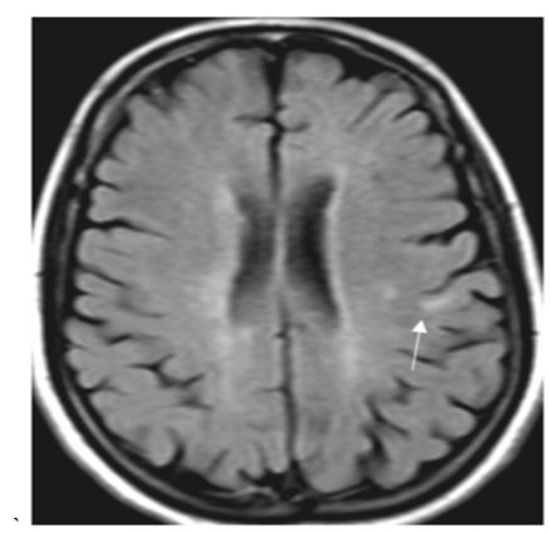

Fig.6 Axial T2/FLAIR of case (2). MS plaques are most evident at left parietal region (white arrow). 

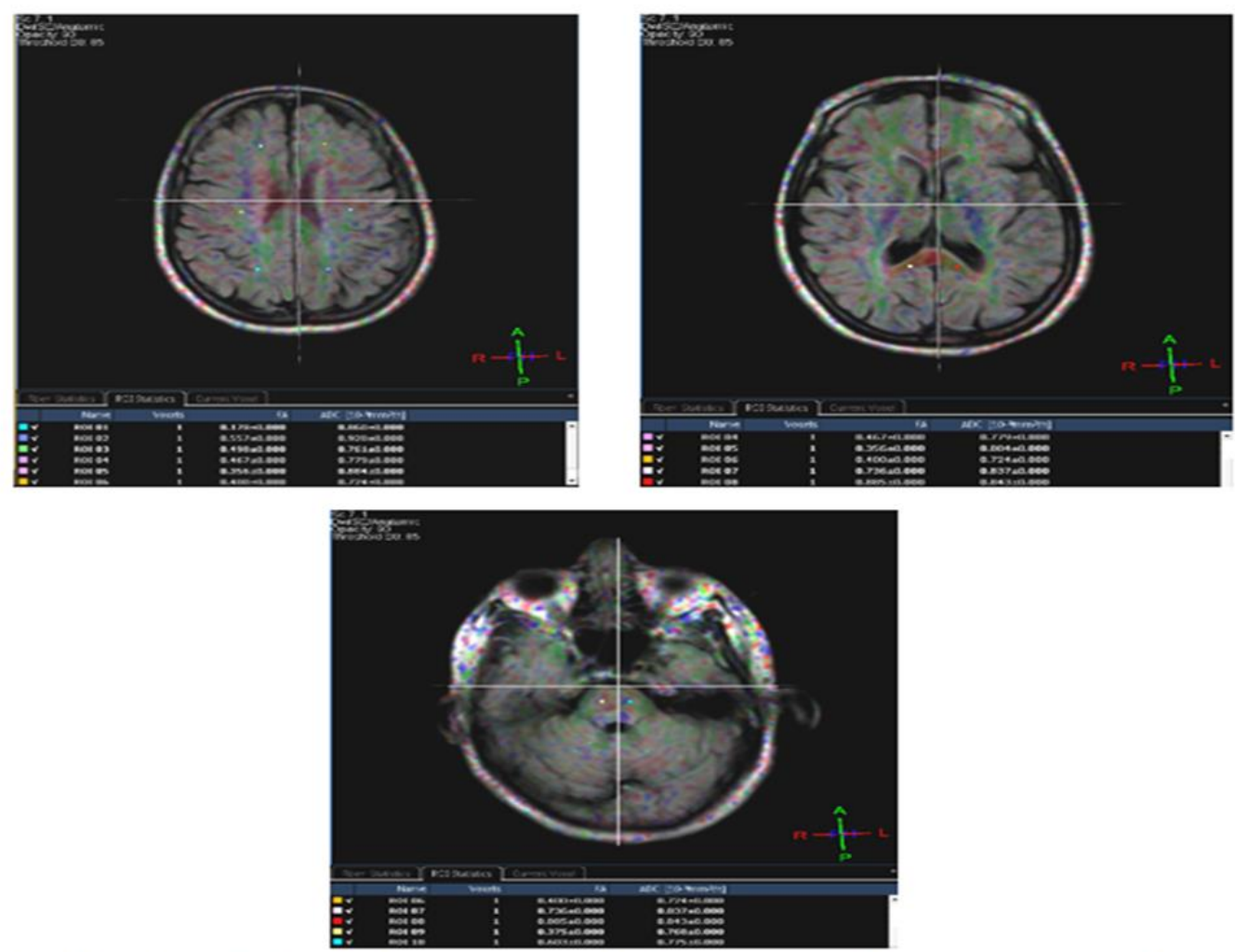

Fig. 7 Fused FLAIR/DTI with multiple ROI at both frontal, parietal, occipital, corpus callosum and brain stem on normally appearing white matter with parametric indices results (FA, MD and $\mathrm{ADC}$ ) in case (2).

Table 2: Parametric indices of case (2)

\begin{tabular}{lccc}
\hline White matter region & FA & ADC & MD \\
\hline Right frontal & 0.40 & 0.9 & 0.0031 \\
Left frontal & 0.40 & 0.8 & 0.0028 \\
Right parietal & 0.50 & 0.8 & 0.0030 \\
Left parietal & 0.50 & 0.8 & 0.0032 \\
Right occipital & 0.20 & 0.9 & 0.0030 \\
Left occipital & 0.60 & 0.9 & 0.0033 \\
Corpus callosum & 0.75 & 0.8 & 0.0026 \\
Brain stem & 0.40 & 0.8 & 0.0031 \\
\hline
\end{tabular}


Case (3)

- Clinical profile: MS female patient of 21 years old with recurrent bouts of clinical symptoms.

The conventional MRI findings: Irregular patchy areas (plaques) of abnormal white matter signal intensity are seen implicating the cerebral subcortical and periventricular regions. They are seen mostly implicating the right frontoparietal region (Fig 8).
-DTI findings: Fused FLAIR/DTI axial images revealed evident heterogeneous cartography maps colors of the frontoparietal white matter (Fig.9) denoting NAWM affection with parametric indices seen in table. 3 .

- Comment: Significant reduction of the FA values of the NAWM within the frontal regions i.e. the most affected regions on conventional MRI

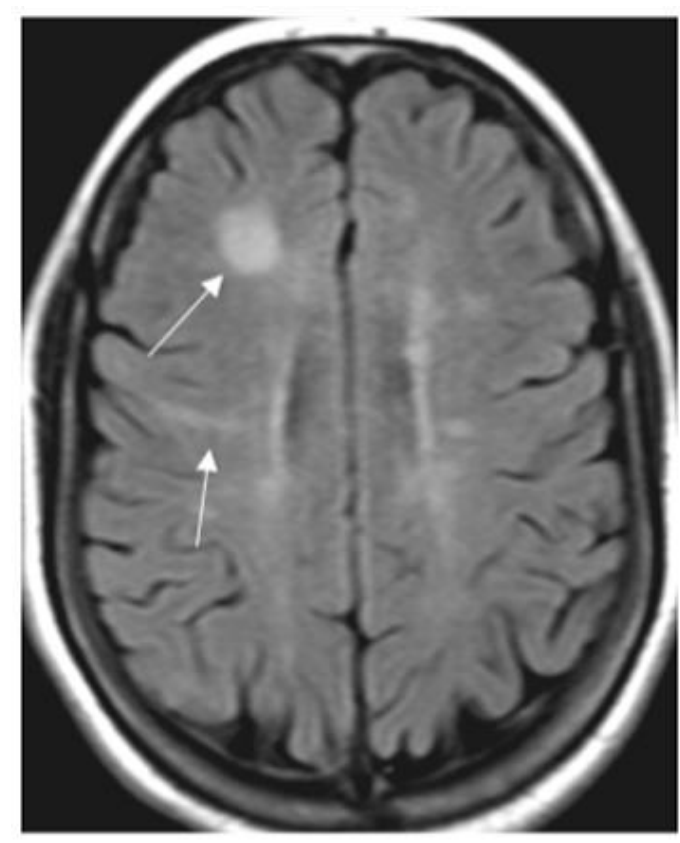

Fig. 8 Axial T2/FLAIR of case (3). MS plaques are most evident at right frontoparietal region (white arrows). 

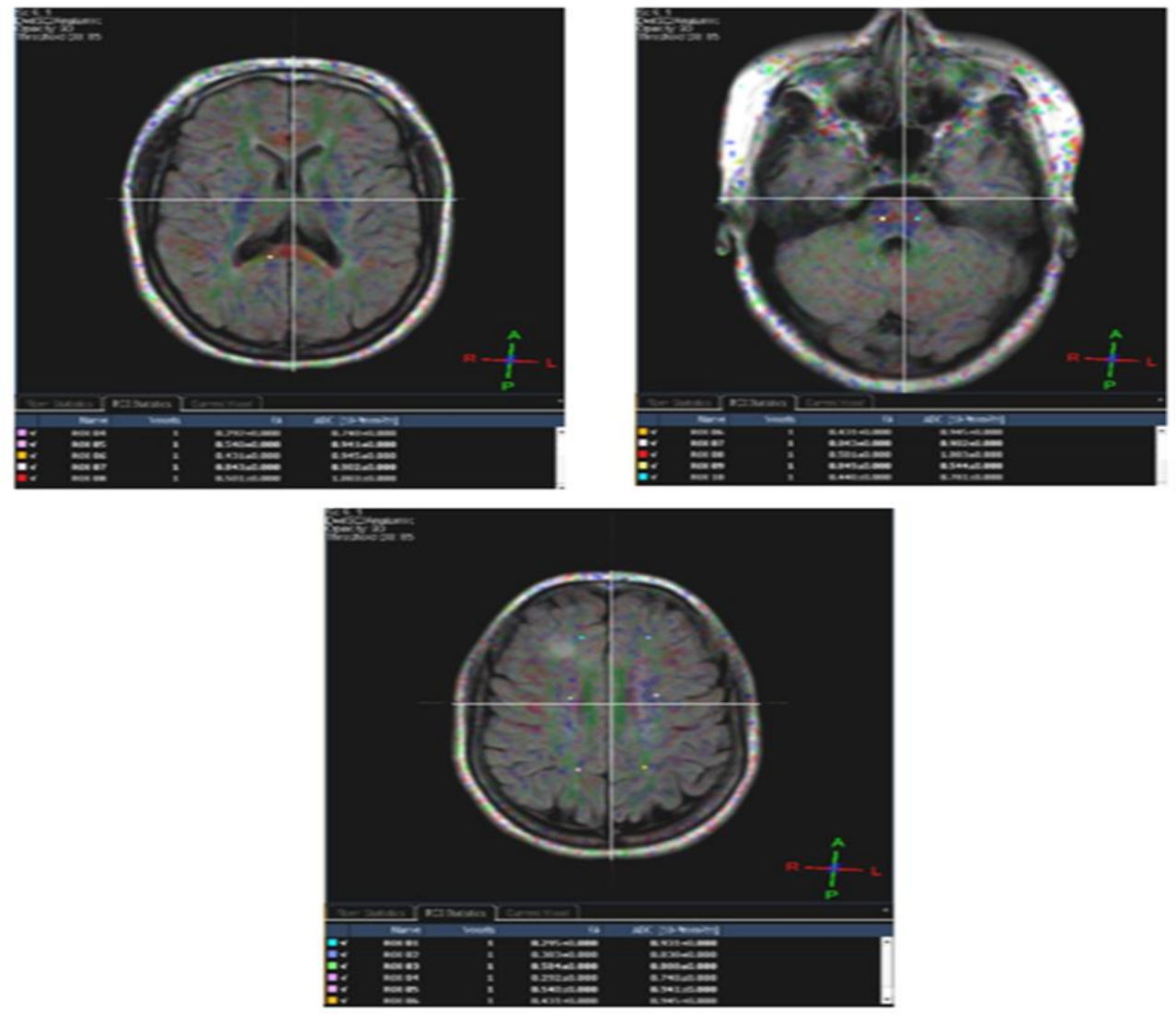

Fig. 9 Fused FLAIR/DTI with multiple ROI at both frontal, parietal, occipital, corpus callosum and brain stem on normally appearing white matter with parametric indices results (FA, MD and ADC) in case (3).

Table 3: Parametric indices of case (3)

\begin{tabular}{llll}
\hline White matter region & FA & ADC & MD \\
\hline Right frontal & 0.30 & 0.9 & 0.0030 \\
Left frontal & 0.30 & 0.6 & 0.0026 \\
Right parietal & 0.60 & 0.9 & 0.0032 \\
Left parietal & 0.30 & 0.7 & 0.0028 \\
Right occipital & 0.50 & 0.9 & 0.0025 \\
Left occipital & 0.40 & 0.9 & 0.0027 \\
Corpus callosum & 0.64 & 0.9 & 0.0033 \\
Brain stem & 0.60 & 0.7 & 0.0031 \\
\hline
\end{tabular}




\section{Discussion}

MS is a chronic inflammatory-demyelinating and neurodegenerative disease of the CNS and is the commonest cause of non-traumatic debility in young and middle-age adults ${ }^{(3)}$.

Pathologically, MS is characterized by areas of demyelinated plaques dispersed throughout the MS lesions reveal an excessive heterogeneity with respect to the presence and extent of inflammation, demyelination, axonal injury, gliosis and remyelination $^{(4)}$.

Diagnosis of MS is based on clinical findings; however, MRI is integrated in the diagnostic criteria of the disease as a result of its unique sensitivity in demonstrating DIS and DIT of demyelinating lesions in the brain (5).

In spite of its increasing role in clinical management and scientific investigation of MS, conventional MRI is limited by low pathological specificity and low sensitivity to diffuse damage in NAWM. Conventional MRI shows only limited associations with clinical status as well ${ }^{(6)}$.

DTI is a quantitative technique that is able to overcome those limitations by providing more specificity to the underlying pathologic substrates of the disease and more sensitive to the full extent of 'occult' tissue destruction in patients with MS. Diffusion depends on measuring the microscopic Brownian motion of water molecules ${ }^{(7)}$.

In our study, NAWM of the brain seen on conventional MR in 50 patients with MS was examined using DTI and the data was compared to the findings in normal age and sex matched control subjects. We examined changes in MD, FA and ADC and correlated these parametric indices to each other and to the morphologic changes detected on conventional MRI and related to disease severity.

In our study, we found that $40 \%$ of the examined cases showed increased MD in what looked like NAWM indicating the presence of structural damage beyond the level of morphologic appearance.

In our study, we also examined the relation between the degree of $F A$ reduction in NAWM and its relation to the location of the largest MS plaque. In agreement with Peterson et al. ${ }^{(6)}$, our study showed that regions of maximum $\mathrm{FA}$ reduction of the NAWM are not always related to the largest 
demyelinating plaque seen on conventional MRI. This indicates that significant heterogeneity in the damaging processes acting in NAWM has no relation to sites of established or more severe injury, even within the same brain. This means that the process of white matter damage is not simply a retrograde neuronal degeneration going in line with areas of severe morphologic damage.

In our study $84 \%$ of the examined patients showed at least one region of reduced FA of the NAWM while only $40 \%$ showed increase in the mean diffusivity. This proves that pathological changes responsible for the change noted in either parameter are not the same and that FA is likely a more sensitive indicator for underlying the NAWM damage.

In our study, we also examined the relation between the changes in FA values and the burden of the disease as one of the indicators of disease severity. $20 \%$ of our cases showed more than seven definite demyelinating plaques denoting high burden of the disease, $80 \%$ of these patients showed three or more regions of NAWM FA reduction and 10\% showed two regions of NAWM FA reduction. Among the patients with three or more areas of FA reduction in the NAWM, $73 \%$ showed heavy burden of the disease.
This indicates, that with higher burden of the disease there is higher incidence of NAWM damage and indicate that the DTI examination of NAWM can play an important role in monitoring not only morphologic severity but also occult damage extent.

Prospective studies are warranted to precise the accuracy of DTI in detecting longitudinal MS-related changes. This issue is cornerstone for future application of DTI to the monitoring of the disease evolution in MS clinical trials and, eventually, in the assessment of individual patients ${ }^{(7)}$.

The main limitation of tractography studies in MS are those that are generally found in all tractography studies. The main limitation of DTI studies in MS is that the exact pathological substrates of in vivo diffusion changes are not known. Therefore, studies investigating radiological and pathological parameters are needed to understand the underlying tissue abnormalities ${ }^{(8)}$.

Our study has some other potential limitation such as low number of participants, geographical limitation of the participating patients, refusal of some patients to take part in our study, difficulty in prolonged follow up of MRI changes as well as lack of longitudinal clinical correlation with DT 
changes. Other studies have reported significant correlation between DTI findings and MS clinical symptoms and disability and suggested a role for DTI as predictive indicator of clinical outcome and as a tool for monitoring response to treatment in clinical trials; however in our study we couldn't examine the clinical correlates of DTI changes in NAWM.

Further studies are needed to further assess the possibility of presence of a relation between the sites of predilection of MS parenchymal changes and the clinical presentation and between the sites of predilection of MS parenchymal changes and the disease subtype and course.

\section{Conclusion}

We showed that DTI technique can be used as base line study to assess the disease severity and distribution in NAWM. Further studies for the clinical correlation, longitudinal follow up and the assessment of treatment efficacy is highly indicated for fully establishing the role of DTI examination of the NAWM in diagnosis and management of patients with MS.

\section{References}

1- McDonald WI, Compston A. \& Edan G. (2001) Recommended diagnostic criteria for multiple sclerosis: guidelines from the international panel on the diagnosis of multiple sclerosis. Ann Neurol; 50:121-127.

2- Filippi M, Rocca MA, Ciccarelli O, et al. (2016) MRI criteria for the diagnosis of multiple sclerosis: MAGNIMS consensus guidelines. Lancet Neurol 2016; 15: 292-303.

3- Enzinger $\mathrm{C}$ et al (2015) Nonconventional MRI and microstructural cerebral changes in multiple sclerosis. Nat Rev Neurol 11(12):676-686

4- Lassmann H, Bruck W \& Lucchinetti C. (2011) Heterogeneity of multiple sclerosis pathogenesis: implications for diagnosis and therapy. Trends Mol Med. 2011; 7(3):115-121.

5- Le BihanD. (2013) Looking into the functional architecture of the brain with diffusion MRI. Nat Rev Neurosci 2013; 4: 469-480.

6- Peterson JW, Bo L \& Mork S. (2011) Transected neurites, apoptotic neurons, and reduced inflammation in cortical multiple sclerosis lesions. Ann Neurol. 2011; 50(3):389-400.

7- Agosta F, Benedetti B \& Rocca MA. Quantification of cervical cord pathology in primary progressive MS using diffusion tensor MRI. Neurology. 2009; 64(4):631-635.

8- Basser PJ, Mattiello J \& LeBihan D. (2004) MR diffusion tensor spectroscopy and imaging. Biophys J1 2004; 66: 259-267.

To cite this article: Hesham E. El Sheikh, Ahmed S. Mohamed, Ehab M. Sharweda. Role of Magnetic Resonance Imaging with Diffusion Sequences In Assessment of Multiple Sclerosis in Brain Versus Normally Appearing White Matter. BMFJ 2021; 38 (Radiology): 207-219. DOI: 10.21608/bmfj.2020.30033.1262 